\title{
The Research on Dynamic Infrared Scene Simulation for Infrared Seeker
}

\author{
Bo Yang ${ }^{a,}{ }^{*}$ and Ming Lv ${ }^{b}$
}

College of Mechatronic Engineering and Automating, National University of Defense Technology, Changsha 410073, China.

a1023530047@qq.com, bnudtIm@163.com

Keywords: Infrared Scene; Vega; Dynamic; Simulation.

\begin{abstract}
In the field of performance evaluation for infrared guidance system, the dynamic infrared scene simulation has important application value. In order to get a realistic and practical dynamic infrared image, the dynamic simulation is carried out based on Vega and its infrared module. The target and terrain geometry model is created. The infrared characteristic of the thermal dynamic tank and complex terrain is analyzed. The infrared radiation model is established. Using the tools of Vega, the material is mapped and the atmospheric transmission data is calculated. The internal heat source is simulated by editing the object temperature. Through the infrared scene simulation, the high timeefficiency dynamic infrared image comes into being. The results show that the dynamic infrared scene based on Vega can be applied to the performance evaluation of infrared guidance system. At the same time, it also provides a software platform to the semi-physical simulation system which used by testing the guidance system performance.
\end{abstract}

\section{Introduction}

Infrared guidance mode is one of the important guidance methods for various missiles. Infrared scene simulation can be used to simulate the image of infrared seeker in various battlefield environment, which is helpful to evaluate the performance of infrared guidance system. Compared with the method of live shooting, the method of infrared scene simulation can shorten the development period, and reduce the cost of research and development.

In foreign countries, the technology of infrared scene simulation started earlier than china. It has been very mature. There are so many scene simulation systems with infrared modules, such as Vega Prime. In recent years, our researchers have done a lot of research on infrared scene simulation. Wang Xuewei et al. ${ }^{1}$, by solving the temperature field and infrared radiation data, use OpenGL shading language to simulate the dy namic infrared scene of the aircraft. Wang Yan et al. ${ }^{2,3,4}$ done the infrared scene simulation of the ocean, using normal maps and GPU shader technology. Cui Wenyu et al. study on the infrared imaging simulation of complex terrain ${ }^{5}$.

In this paper, based on the Vega platform and its infrared module, the infrared scene simulation of the typical ground scene and the moving target is carried out. Some highly efficient and realistic infrared image have been acquired. This paper provides a theoretical and practical basis for the further simulation of the infrared image of missile attack, and the hardware-in-the-loop simulation system which is used to test the missile guidance system performance.

\section{Geometric Modeling}

\subsection{Establishment of Target Model}

For battlefield real-time roaming and infrared simulation, we must ensure the authenticity of the model, as well as the real-time performance of the simulation. This requires the establishment of the target and background geometry model, under the condition of without excessive distortion, to minimize the model number.

MultiGen Creator is an important tool for virtual reality system modeling. It is able to generate models with a LOD hierarchy of nodes. These models can be directly imported into Vega, OSG and other visual simulation platform. This data format can also greatly improve the efficiency of visual 
platform in infrared simulation. In this paper, the M1A2 main battle tank is selected as the object of infrared simulation. We use MultiGen Creator 4.2 to model.

The main parameters of M1A2: a total length of 9.830 meters, 3.658 meters wide, 0.492 meters from the ground floor, $120 \mathrm{~mm}$ smoothbore gun. In the process of modeling, simple and complex is moderate. At the same time, the LOD modeling technique is used to automatically adjust the fine degree of the surface when the observation position is far away. After the creation of the model structure, in order to effectively improve the sense of reality, we use the model of texture, to make the model look more realistic. At the same time, in the infrared simulation, the visible light texture can be mapped into the material to reflect the infrared radiation emissivity. As shown in Fig. 1, (a) is a simplified model, (b) is a fine model.

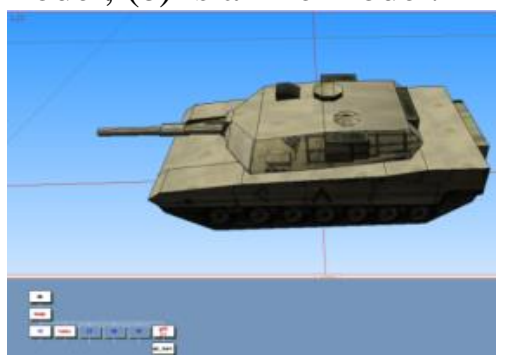

(a)

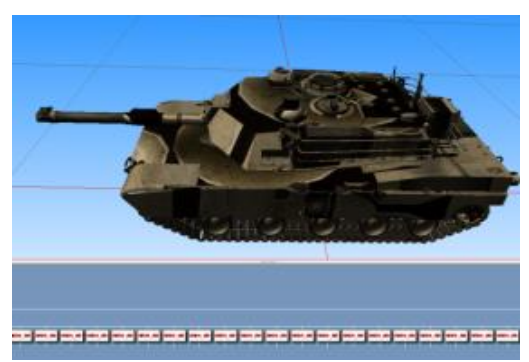

(b)

\subsection{Establishment of Terrain Model}

Figure 1. The model of tank

There are many modeling methods and tools of the terrain, including MultiGen Creator, Terra Vista and VPB tools in OSG. In addition, we can use oblique photography technology to establish the models.

In this paper, Terra Vista 6.2 is used to model the terrain. The terrain of the nature is complex. The modeling process needs to be properly simplified. In this study, the elevation data and the satellite remote sensing data were imported into Terra Vista. Besides, we can also add a number of other types of data, including cultural data, other models, texture maps, etc. After setting the relevant parameters, a more real terrain model can be exported. As shown in Fig.2:

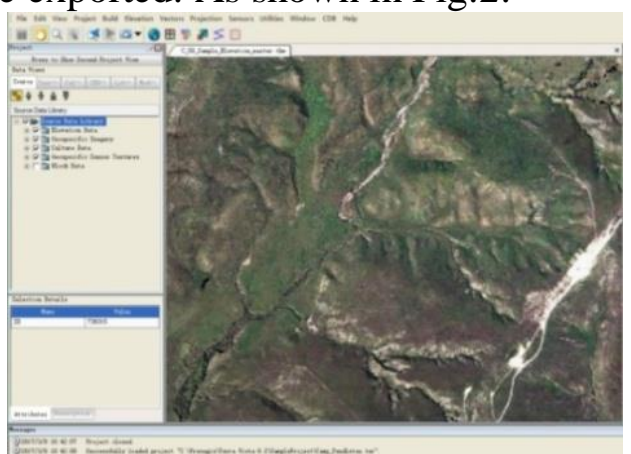

Figure 2. Create terrain with Terra Vista

The generated terrain is composed of triangular meshes. Compared with other quadrilateral, such as pentagon shape mesh, triangular mesh can simulate complex terrain. Each vertex of the triangular mesh corresponds to the adjacent pixels of elevation data. The satellite images are pasted on the terrain in the form of texture to improve the reality of terrain.

\section{Infrared Radiation Model}

According to the heat transfer performance of the object, it is necessary to analyze the infrared characteristics of the target and background. Because of the complex terrain, there is a great amount of surface elements in the whole simulation scene. Then, it will greatly affect the timeliness of the infrared simulation. In order to obtain the physical model of heat transfer and heat radiation which is easy to be processed, the three-dimensional heat transfer model is simplified. One-dimensional heat transfer model has been used. In view of the low heat flux in the horizontal direction of the terrain surface, it is more reasonable to use one-dimensional heat transfer model. 
According to the one-dimensional heat transfer model, the heat transfer equation of the surface of the object is:

$$
\rho c_{P} \frac{d T}{d t}=Q
$$

In this formula, $\rho$ is density of the surface material. $c_{P}$ is specific heat of the material. $T$ is surface temperature. $t$ is time. $Q$ is the sum of receiving and radiating heat. The main considerations include the surface and its internal heat conduction, surface and atmospheric heat exchange, the solar radiation energy and other factors.

In the process of dynamic infrared simulation, the related factors is:

(1) Time and Place

They determine the zenith angle of the sun and the solar radiation constant.

(2) Temperature

(3) Cloud Parameters

It determines cloud radiation.

(4) Speed of Wind

The magnitude of the wind speed effects the heat conduction and the surface temperature of the model.

(5) Observer Altitudes

It determines the position of the viewpoint, the composition of the infrared image and the atmospheric scattering in the infrared radiative transfer.

(6) Infrared Band

The main observation band of infrared detector is 3-5 micron and 8-14 micron. The infrared radiation brightness of the same object in different bands is different.

(7) Internal Heat Source

In the state of motion or shooting, the temperature of the tank surface will vary greatly. Especially after multiple rounds of firing, the temperature of the gun will be very high. It can reach $180 \mathrm{oC} 6$. At the same time, other objects with internal heat source should be considered.

The infrared radiation includes spontaneous radiation and reflected radiation. In addition, it is necessary to consider the influence of atmospheric transmission. The influence of atmosphere on infrared system is mainly reflected in the radiation attenuation. In the simulation, the atmospheric transmission characteristics of solar and lunar radiation, spontaneous emission and infrared radiation are considered. The infrared radiation received by the infrared seeker can be expressed as a simplified formula:

$$
L_{\text {observer }}=\left(\delta L_{s} \cos \theta+L_{\text {object }}\right) T_{\text {path }}
$$

In this formula, $L_{\text {observer }}$ is the radiation received by the seeker, $\delta$ is the material surface reflectance, $L_{s}$ is the radiation of the sun or moon, $\theta$ is the angle between the surface normal and the line from the object to the sun, $T_{\text {path }}$ is the transmittance on the radiative transfer path. $L_{\text {object }}$ is the spontaneous emission.

According to the Planck formula, the formula of radiant brightness is:

$L=\frac{1}{\lambda^{5}} \frac{2 h c^{2}}{e^{h c / \lambda k t}-1}$

In this formula, Planck parameter is $h=6.63 \times 10^{-34} \mathrm{~J} \cdot S, k=1.38 \times 10^{-23} \mathrm{~J} \cdot K^{-1}, \lambda$ is the wavelength. Infrared simulation is selected in 3-5 micron. The spontaneous emission $L_{o b j e c t}$ can be obtained by integrating $L$ in the range of 3-5 micron.

There are three states of tanks: cold static state, thermal static state and thermal dynamic state. In the thermal dynamic state of the tank, the tank is in a state of motion. The factors that need to be taken into account, includ the track transmission and movement speed and other factors 7 . The calculation of temperature field is needed to solve the radiation. Firstly, the tank model is divided into many triangular meshes. Secondly, the temperature field is calculated by software Fluent. Thirdly, 
according to the Planck formula, the distribution of thermal radiation is solved. In the infrared scene simulation of the attacking missile, it is not necessary to make the temperature distribution too fine.

\section{Scene Simulation}

Vega is a software platform for developing interactive and visual simulation applications. With its infrared module, it can be used for material mapping and atmospheric environment database generation.

\subsection{Material Mapping}

The radiant reflectivity of the surface is mainly affected by the material. The visible light texture of the object need to map into the material texture. The object material is pasted on the surface of the model in the form of texture. Then, the infrared module SensorVision will conveniently read in the infrared simulation process.

Texture Material Mapper (TMM) in Vage can be used to achieve this function. The TMM tool has a hundred kinds of materials, including soil, Rock, pure, vegetation, building materials, synthetic materials etc. ${ }^{8}$ Each material corresponds to its thermal reflectivity. After the texture of the model is imported in TMM, we use the supplied selection, Lasso, or magic wand tool to select the area to specify the material. So that, the material properties are selected for each pixel. After the material texture is generated, it is saved in the format of tmm. As shown in Fig.3:

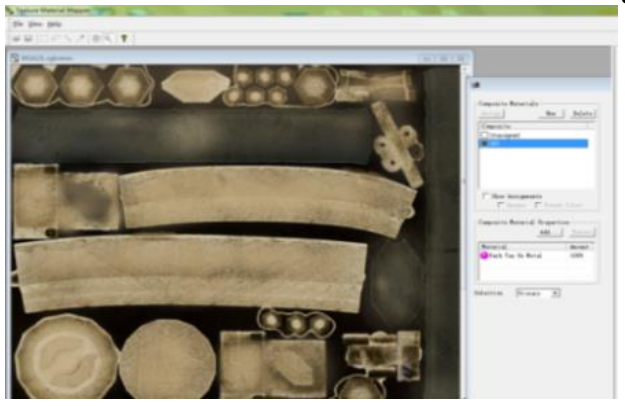

\subsection{Air Data Calculation}

Figure 3. Material Mapping

Atmospheric transmission simulation softwares commonly used are LOWTRAN, MODTRAN, FASCODE, and so on. In Vega, we can use its Mosart Atmospheric Tool (MAT) to finish the job. It can be used to calculate the atmospheric transmittance, the atmospheric background radiation, the direct radiation of the sun and the moon.

Using this tool, we can get multiple states data at a time.

We can set a variety of parameters on the simulation. For example, geographical location, time, weather, clouds, humidity, wind speed, temperature, the location of the observer, the target azimuth, the sun position, infrared wavelength, heat transfer, and so on. As shown in Fig.4 is the temperature variation curve of one material in the 24 hours a day.

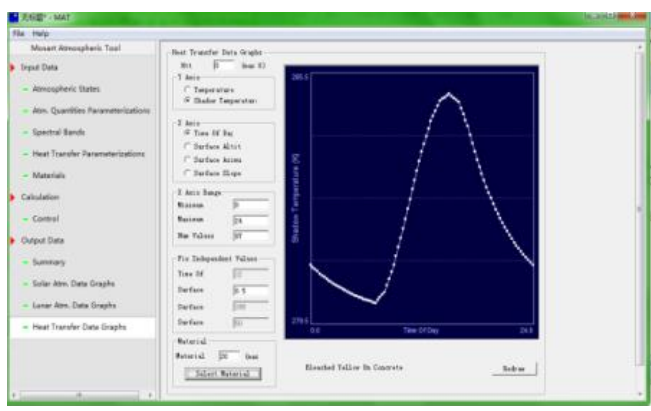

Figure 4. Temperature variation curve of one material

\subsection{Setting the Simulation Environment}

The kinetic friction, the engine, and the shooting will bring about the temperature of the tank surface rise. In this paper, the tank has gotten high-speed shooting just now. As well as, the tank is 
moving. We use the "object property editor" option to edit the temperature which will simulate the internal heat. As shown in Fig.5 is the editing interface of temperature.

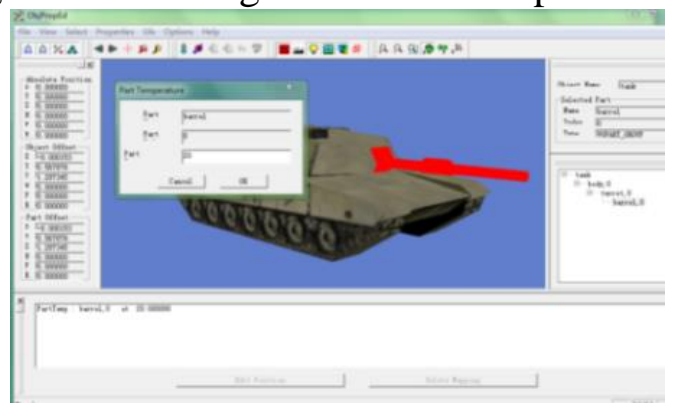

Figure 5. Edit model properties

In order to simulate the state of the tank, the "pathing" and "navigators" modules are used to set the path of the tank. At the same time, the speed of the tank is variable. Fig.6 shows how to set the motion path of the tank.

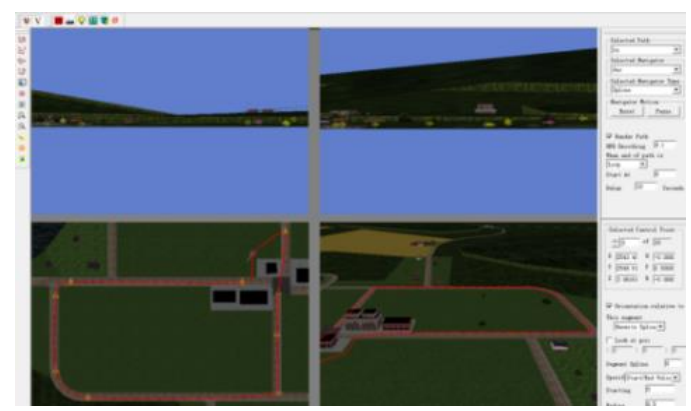

Figure 6. Set the athletic path

Before the simulation, the .mat file need be put into the Database Manager module of Vega.

\subsection{Result of Simulation}

The simulation conditions is: The position is in 30 degrees north latitude, 120 degrees east longitude. Time is March 3, 2017. Cloud is clear. Temperature is 27 degrees. Wavelength range is in the 3-5 micron. Wavelength response coefficient is 1 . The tank is in a state of continuous firing. The infrared image is shown in Fig.7.

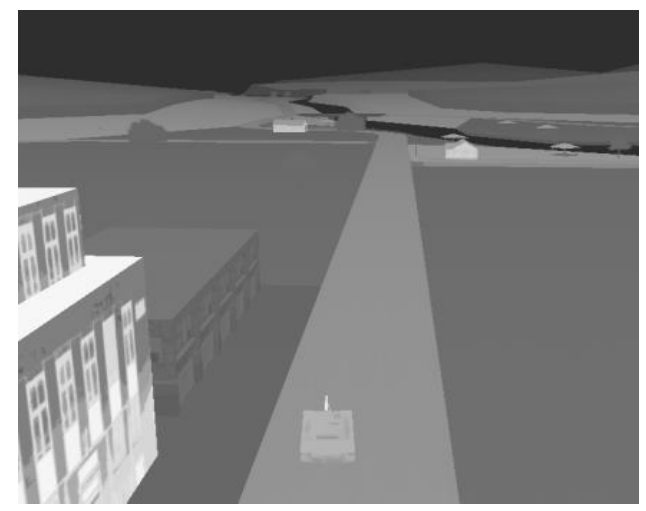

Figure 7. Infrared simulation image

Due to the influence of atmospheric transmission and image processing module, the infrared image of the missile will have a lot of noise. SensorWorks module can be used to simulate a number of typical sensor effects, such as platform jitter, random noise, etc. It will bring a more realistic simulation. In the process of infrared simulation, we have added the random noise signal. The infrared simulation image is shown in Fig.8. 


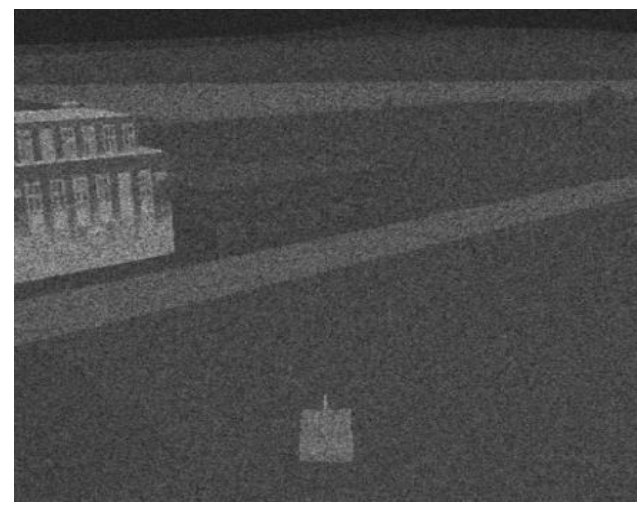

Figure 8. Noisy image

By using the serial communication tool, the missile position and attitude data can be imported into the simulation platform. The observation position will be changed along with the missile. So that, we can simulation the process of attack.

\section{Conclusion}

In this paper, based on Vega and its infrared module, the dynamic infrared scene simulation is carried out for the tank in motion. The modeling and simulation method of infrared scene is studied. The results show that the simulation results are good. The research will be used to test the performance of missile guidance system in real time. It will provide the necessary experimental data for the improvement of the guidance system. As Vega is a mature commercial software, its functions have been encapsulated. So if we want to do further simulation research, it is necessary to use its API function.

At the same time, this study also provides an important reference for the further research on the open source scene simulation platform. It is a key step to build a new and more open infrared scene simulation platform.

\section{Acknowledgments}

Yang Bo, male, born in 1990, Han nationality, Master Degree Candidate, study on Precision Guidance and Control. My tutor, Lv Ming, male, born in 1957, Senior Engineer, currently teaching at the College of Mechatronic Engineering and Automating National University of Defense Technology, Master's tutor. Thanks to my tutor Lv Ming and other teachers and students in our lab for patient help. With their help, I can only finish my paper efficiently. In addition, I deeply appreciate the contribution to this thesis made in various ways by my friends and classmates.

\section{References}

[1]. Wang Xuewei and Zhang Weiguo. "Computer generation of dynamic infrared images of a plane", Infrared and laser Engineering, 1999, 28(4), pp.21-24.

[2]. Wang Yan, Xie Xiaofang, Sun Haiwen, et al. "Simulation of dynamic infrared sea scene in mid band", Infrared and laser Engineering, 2015, 44(12), pp.3604-3608.

[3]. Wang Yan, Xie Xiaofang, Yang Jian, et al. "Research on Scene simulation of IR seeker and Its implementation based on OSG", Science Technology and Engineering, 2015, 15(16), pp.186189.

[4]. Wang Yan, Xie Xiaofang, Yang Jian, et al. "The research on dynamic simulation of IR sea background scene", Journal of Projectiles, Rockets, Missiles and Guidance, 2015, 35(5), pp.199201.

[5]. Cui Wenyu, Yi Weining, Qiao Yanli, et al. "Thermal infrared imaging simulation for complex terrain”, Infrared and laser Engineering, 2014, 43(10), pp.3206-3210.

[6]. Luo LaiKe, Xuan Yimin, and Han Yuge. "Finite element calculation of temperature field of tank gun barrel”, Acta Armamentarii, 2005, 26(1), pp.7-8. 
[7]. Han Yuge, Cheng Zhiduo, Ren Dengfeng, et al. "Thermal interactions and infrared simulation of armored vehicles and ground background", Infrared and laser Engineering, 2013, 42(1), pp.2025.

[8]. Wang Jie. "Research on real time walkthrough and infrared simulation of Virtual Battlefield Based on VR Technology", Nanjing University of Science and Technology, 2013, pp.26-33.

[9]. Zhou Fan. "Research on Dynamic Simulation of infrared scene of ground target and background based on VEGA", Nanjing University of Science and Technology, 2016, pp.24-32. 